\title{
Qualities of a vibrator, the elastic element of which has different coefficients of stiffness in the two intervals of displacements, in a separate case
}

\author{
K. Ragulskis ${ }^{1}$, B. Spruogis ${ }^{2}$, M. Bogdevičius ${ }^{3}$, A. Matuliauskas ${ }^{4}$, V. Mištinas ${ }^{5}$, L. Ragulskis ${ }^{6}$ \\ ${ }^{1}$ Kaunas University of Technology, K. Donelaičio Str. 73, LT-44249, Kaunas, Lithuania \\ 2,3,4,5 Department of Mobile Machinery and Railway Transport, Faculty of Transport Engineering, Vilnius \\ Gediminas Technical University, Plytinès Str. 27, LT-10105, Vilnius, Lithuania \\ ${ }^{6}$ Department of Systems Analysis, Faculty of Informatics, Vytautas Magnus University, Vileikos Str. 8, \\ LT-44404, Kaunas, Lithuania \\ ${ }^{1}$ Corresponding author \\ E-mail: ${ }^{1}$ kazimieras3@hotmail.com, ${ }^{2}$ bronislovas.spruogis@gmail.com, ${ }^{3}$ marijonas.bogdevicius@vgtu.lt, \\ ${ }^{4}$ arvydas.matuliauskas@vgtu.lt, ${ }^{5}$ vygantas.mistinas@gmail.com, ${ }^{6}$.ragulskis@if.vdu.lt
}

Received 21 April 2020; received in revised form 4 June 2020; accepted 13 June 2020

DOI https://doi.org/10.21595/jmeacs.2020.21532

Check for updates

Copyright $(2020$ K. Ragulskis, et al. This is an open access article distributed under the Creative Commons Attribution License, which permits unrestricted use, distribution, and reproduction in any medium, provided the original work is properly cited.

\begin{abstract}
Generators of mechanical vibrations with nonlinear stiffness of the elastic element in the conservative case are investigated. It is shown that the vibrator of this type generates several harmonic components and that the number of those multiple harmonics can be controlled. One period of motion is investigated. The presented analytical relationships and numerically obtained graphical relationships reveal the qualities of the system and enable to choose the desirable parameters of motions. The performed investigation of this system showed that eigenvibrations take place with linear spectrums when the system in separate intervals consists from two linear parts. This takes place in the case when the border of difference of coefficients of stiffness is located in the position of equilibrium of the system. In both intervals vibrations by separate partial frequencies take place and general motion depends on the eigenfrequency of the whole system. Of course the latter frequency depends on both partial frequencies. General motion of the whole system takes place according to the infinite linear spectrum of eigenfrequencies. All this enables to create enhanced vibrators by using those qualities and to use them in technologies.
\end{abstract}

Keywords: mechanical generators of vibrations, conservative system, harmonic excitation, nonlinear qualities.

\section{Introduction}

In a number of publications basis of the theory of nonlinear mechanical generators of vibrations were investigated as well as their multi valued steady state regimes. The distinctive feature of this research work is in the fact that they can generate several frequencies under excitation of a single harmonic as well as ensure stable operation in the resonance zones.

This paper is based on the investigations of essentially nonlinear systems and their dynamics presented in a book by Ragulskienè V. Vibro-Shock Systems (Theory and Applications). Vilnius: Mintis, 1974, (in Russian), where change of the jump type in the characteristic of impact velocity which takes place at the point of transition between soft and hard excitations of vibrations is seen. But the essential features are not revealed. This quality was also observed when exciting vibrations of vibromotors in a book by Ragulskis K., Bansevičius R., Barauskas R., Kulvietis G. Vibromotors for Precision Microrobots. New York: Hemisphere, 1987. Here also attention was not directed to this quality. Papers presented in the references were useful for investigations. Nonlinear vibrating systems and their resonances are analysed in [1]. Periodic and transient excitations and the dynamics of impacts are investigated in [2]. Periodic nonlinear systems as well as their stabilization are analysed in [3]. Mechanical systems when impacts take place are investigated in [4]. Mechanical systems, impacts and their periodic orbits are described in [5]. Nonlinear 
vibro-impact energy sink is analysed in [6]. Impact of particle with a wall is investigated in [7]. Determination of frequencies of a mechanical system is analysed in [8]. Mechanism with a pendulum is described and investigated in [9]. Vibrations of a model having piecewise linearity are analysed in [10]. Resonant zones of a dynamical system are investigated in [11]. Investigation of the Sommerfeld effect is performed in [12]. Analysis of isolated resonances of a dynamical system is presented in [13].

Several systems were investigated in which subsystems with different nonlinearities have a point of transition and when this point in the static positions of both subsystems coincides, for example [14]. In this paper the investigation is presented when the quality of this type takes place and the independence of eigenfrequencies from the amplitudes of motions is determined and motion takes place with infinite spectrum of frequencies.

In this paper the system consisting from two subsystems the static positions of equilibrium of which coincide is investigated. The motions are investigated analytically - graphically and they indicate the linearity of spectrums with infinite number of frequencies.

The investigated system is described by the equations of the following type:

$\ddot{x}+\left(p^{2}+p_{0}^{2}\right) x=f \sin (\omega t+\varphi), \quad x=x_{0}$,

$\ddot{x}+p^{2} x=f \sin (\omega t+\varphi), \quad x<x_{0}$

$p_{0}^{2} x_{0}=0, \quad x<x_{0}$,

where $p=\sqrt{C / m}, p_{0}=\sqrt{C_{0} / m}, f=F / m, \omega$ and $\varphi$ are the frequency and phase of excitation respectively, $p$ and $p+p_{0}$ are partial eigenfrequencies of the system.

\section{Conservative system}

Characteristics of the system are determined analytically - numerically when $f=0$.

\subsection{Motion of the system in the first interval}

Investigation of motion of the system in the interval:

$t \in\left[0, T_{0}\right], \quad x=x_{0}$,

is performed.

The following conditions of motion are assumed when:

$t=0, \quad x=x_{0}=0, \quad \dot{x}=\dot{x}^{-}$,

$t=T_{0}, \quad x=x_{0}=0, \quad \dot{x}=-\dot{x}^{-}$.

From the Eq. (1) by taking into account Eqs. (4) and (5) it is obtained:

$$
\begin{aligned}
& x=\frac{\dot{x}^{-}}{\sqrt{p^{2}+p_{0}^{2}}} \sin \sqrt{p^{2}+p_{0}^{2}} t, \\
& \dot{x}=\dot{x}^{-} \cos \sqrt{p^{2}+p_{0}^{2}} t, \\
& \ddot{x}=-\dot{x}^{-} \sqrt{p^{2}+p_{0}^{2}} \sin \sqrt{p^{2}+p_{0}^{2}} t, \\
& \dot{x} \ddot{x}=-0.5\left(\dot{x}^{-}\right)^{2} \sqrt{p^{2}+p_{0}^{2}} \sin 2 \sqrt{p^{2}+p_{0}^{2}} t .
\end{aligned}
$$

From the Eq. (7) by taking into account Eqs. (5) it is obtained: 
$T_{0}=\frac{\pi}{\sqrt{p^{2}+p_{0}^{2}}}$

\subsection{Motion of the system in the second interval}

Investigation of motion of the system in the interval:

$t \in\left[T_{a}, \bar{T}\right]=\left[T_{a}, \frac{2 \pi}{\bar{\omega}}\right], \quad x \leq x_{0}$,

where $\bar{T}$ and $\bar{\omega}$ are the eigenperiod and eigenfrequency of the system respectively, is performed.

At:

$t=\bar{T}, \quad x=x_{0}=0, \quad \dot{x}=\dot{x}^{-}$.

From the Eq. (3) by taking into account Eq. (5) it is obtained:

$x=-\frac{\dot{x}^{-}}{p} \sin p\left(t-T_{0}\right)$,
$\dot{x}=-\dot{x}^{-} \cos p\left(t-T_{0}\right)$,
$\ddot{x}=\dot{x}^{-} p \sin p\left(t-T_{0}\right)$,
$\dot{x} \ddot{x}=-0.5\left(\dot{x}^{-}\right)^{2} p \sin 2 p\left(t-T_{0}\right)$.

From the Eq. (13) by taking into account Eqs. (10) and (12) it is obtained:

$\bar{T}=\frac{\pi}{\sqrt{p^{2}+p_{0}^{2}}}+\frac{\pi}{p} \quad$ or $\quad \bar{T}=\frac{\pi}{p}\left[1+\frac{1}{\sqrt{1+\left(\frac{p_{0}}{p}\right)^{2}}}\right]$.

By taking into account that $\bar{T}=2 \pi / \bar{\omega}$ and on the basis of Eq. (16) it is obtained:

$\bar{\omega}=2 p \frac{\sqrt{1+\left(\frac{p_{0}}{p}\right)^{2}}}{\sqrt{1+\left(\frac{p_{0}}{p}\right)^{2}}+1}$

which is used for the determination of the eigenfrequency as a function of the parameters of the investigated system.

\subsection{Characteristics of the system}

Dynamical characteristics of the system $x, \dot{x}, \ddot{x}, \dot{x} \ddot{x}$ are expanded into the Fourier series with respect to the eigenfrequency.

As an example the expansion of the characteristics of displacement is presented. According to the Eqs. (5) and (13):

$$
x=\frac{\dot{x}^{-}}{p}\left\{\begin{array}{l}
\frac{1}{\sqrt{1+\left(\frac{p_{0}}{p}\right)^{2}}} \sin \sqrt{1+\left(\frac{p_{0}}{p}\right)^{2}} t, \quad 0 \leq t \leq \bar{T}_{0}=\frac{\pi}{p \sqrt{1+\left(\frac{p_{0}}{p}\right)}}, \\
-\sin p\left(t-T_{0}\right), \quad T_{0} \leq t \leq \bar{T},
\end{array}\right.
$$


it is obtained:

$x=\frac{\dot{x}^{-}}{p} \sum_{n=0}^{\infty}\left(a_{n} \sin n \bar{\omega} t+b_{n} \sin n \bar{\omega} t\right)$,

where $a_{n}$ and $b_{n}$ depend only from $p$ and $p_{0} / p$.

In the same way other characteristics are also expanded into the Fourier series:

$$
\begin{aligned}
& \dot{x}=\dot{x}^{-}\left\{\begin{array}{l}
\cos p \sqrt{1+\left(\frac{p_{0}}{p}\right)^{2}} t, \quad 0 \leq t \leq T_{0}, \\
-\cos p\left(t-T_{0}\right), \quad T_{0} \leq t \leq \bar{T},
\end{array}\right. \\
& \ddot{x}=\dot{x}^{-} p\left\{\begin{array}{l}
-\sqrt{1+\left(\frac{p_{0}}{p}\right)^{2}} \sin p \sqrt{1+\left(\frac{p_{0}}{p}\right)^{2}} t, \quad 0 \leq t \leq T_{0}, \\
\sin p\left(t-T_{0}\right), \quad T_{0}<t<\bar{T},
\end{array}\right. \\
& \dot{x} \ddot{x}=-0.5\left(\dot{x}^{-}\right)^{2} p\left\{\begin{array}{l}
\sqrt{p^{2}+\frac{p_{0}}{p}} \sin 2 p \sqrt{p+\left(\frac{p_{0}}{p}\right)^{2}} t, \quad 0 \leq t \leq T_{0}, \\
\sin 2 p\left(t-T_{0}\right),
\end{array}, T_{0}<t<\bar{T} .\right.
\end{aligned}
$$

According to the Eqs. (19) and Eqs. (21-23) their investigation is performed by obtaining graphical relationships $\dot{x}=\dot{x}(x), \ddot{x}=\ddot{x}(\dot{x}), \dot{x} \ddot{x}=\dot{x} \ddot{x}(x) ; x, \dot{x}, \ddot{x}, \dot{x} \ddot{x}$ as functions of time in the period $\bar{T}$ and also the coefficients of their expansion into the Fourier series, that is the graphical representations of eigenfrequencies.

\section{Results of investigations of dynamics of the conservative system}

Graphical results of investigation of dynamics of the system when $p^{2}=1, p_{0}^{2}=1$ are presented in Fig. 1.

Graphical results of investigation of dynamics of the system when $p^{2}=1, p_{0}^{2}=4$ are presented in Fig. 2.

Graphical results of investigation of dynamics of the system when $p^{2}=4, p_{0}^{2}=1$ are presented in Fig. 3.

Amplitude frequency characteristics when $p^{2}=1, p_{0}^{2}=1$ are presented in Fig. 4 .

Amplitude frequency characteristics when $p^{2}=1, p_{0}^{2}=4$ are presented in Fig. 5.

Amplitude frequency characteristics when $p^{2}=4, p_{0}^{2}=1$ are presented in Fig. 6 . 


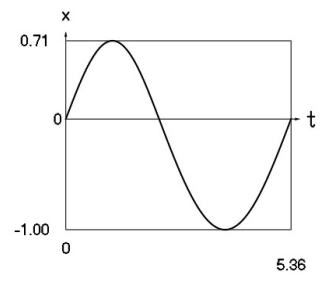

a) Displacement as function of time

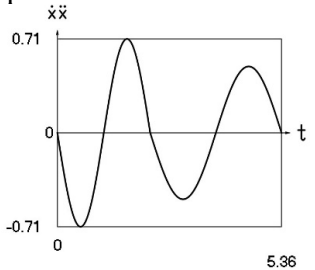

d) Velocity multiplied by acceleration as function of time

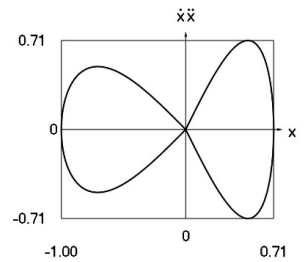

g) Phase trajectory: velocity multiplied by acceleration as function of displacement

Fig. 1. Dynamics of the conservative system when $p^{2}=1, p_{0}^{2}=1$

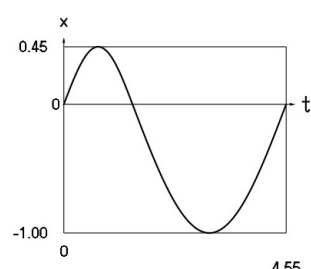

455

a) Displacement as function of time

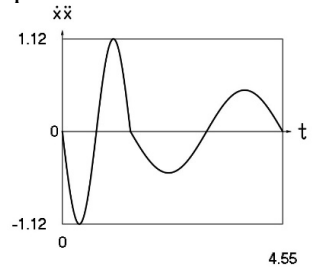

d) Velocity multiplied by acceleration as function of time

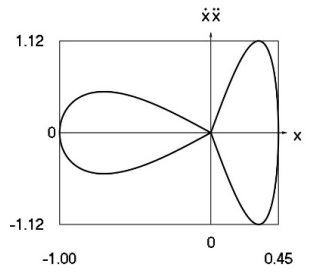

g) Phase trajectory: velocity multiplied by acceleration as function of displacement

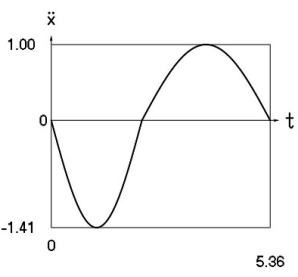

c) Acceleration as function of time

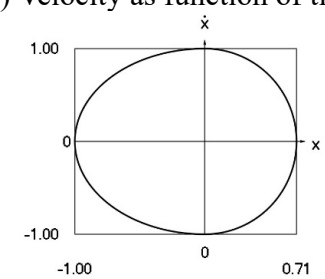

e) Phase trajectory: velocity as function of displacement

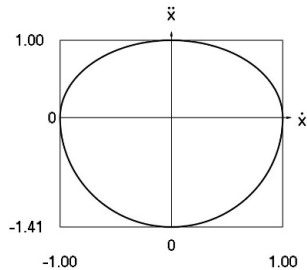

f) Phase trajectory: acceleration as function of velocity

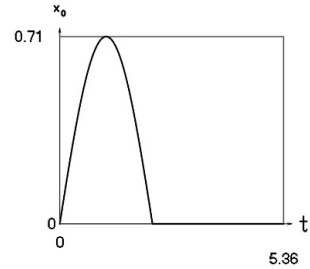

h) Another displacement as function of time 


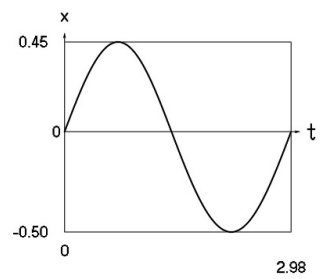

a) Displacement as function of time

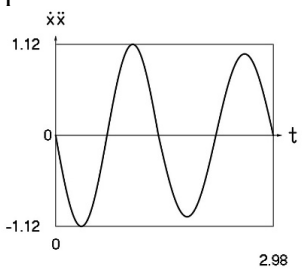

d) Velocity multiplied by acceleration as function of time

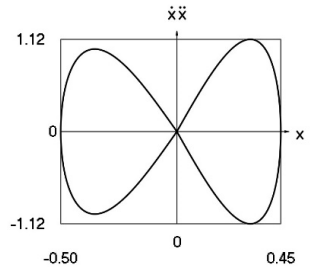

g) Phase trajectory: velocity multiplied by acceleration as function of displacement

Fig. 3. Dynamics of the conservative system when $p^{2}=4, p_{0}^{2}=1$

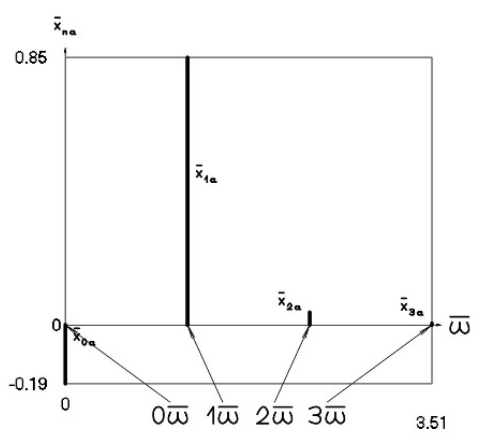

a) Displacement frequency characteristic

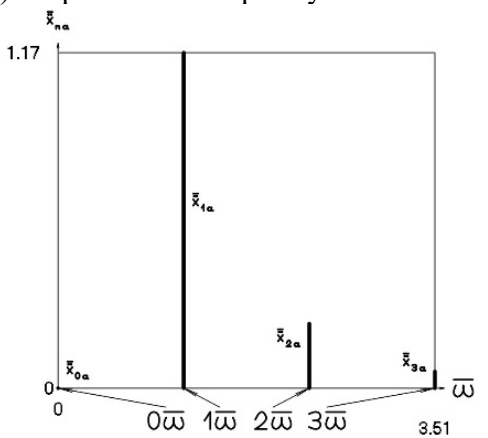

c) Acceleration frequency characteristic

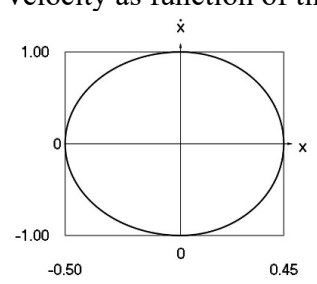

e) Phase trajectory: velocity as function of displacement

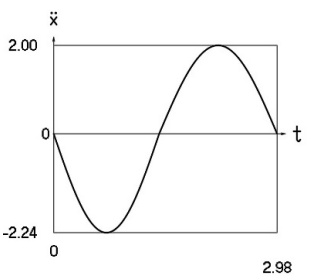

c) Acceleration as function of time

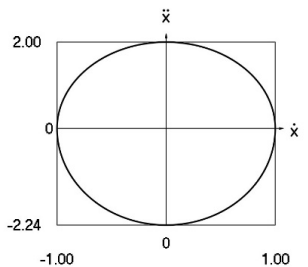

f) Phase trajectory: acceleration as function of velocity

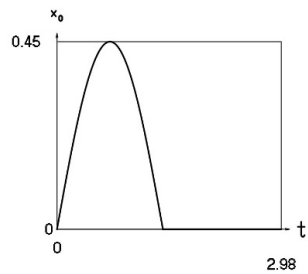

h) Another displacement as function of time 


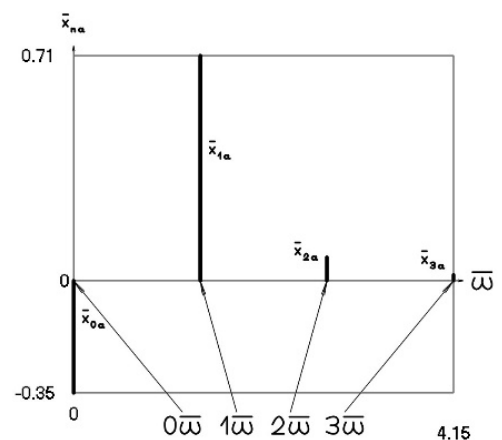

a) Displacement frequency characteristic

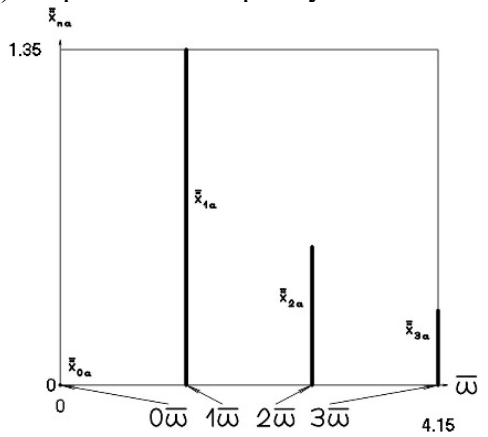

c) Acceleration frequency characteristic

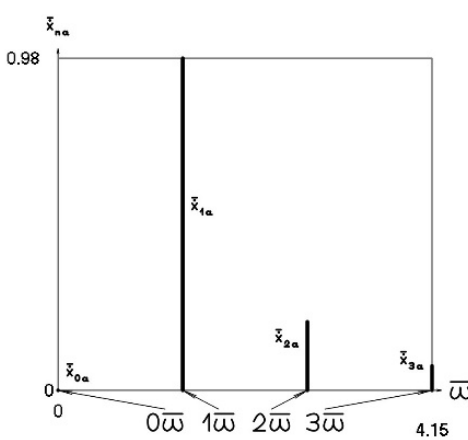

b) Velocity frequency characteristic $\bar{x}_{\mathbf{x}_{\text {na }}}$

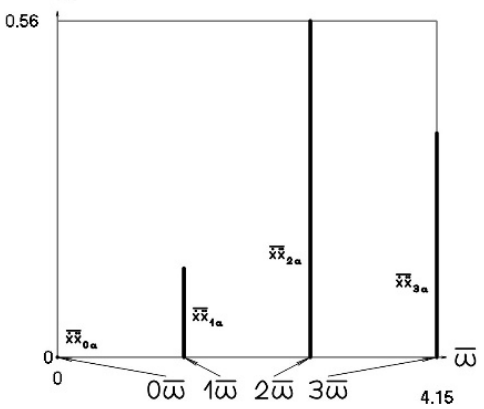

d) Velocity multiplied by acceleration frequency characteristic

Fig. 5. Amplitude frequency characteristics (constant part and first three harmonics) when $p^{2}=1, p_{0}^{2}=4$

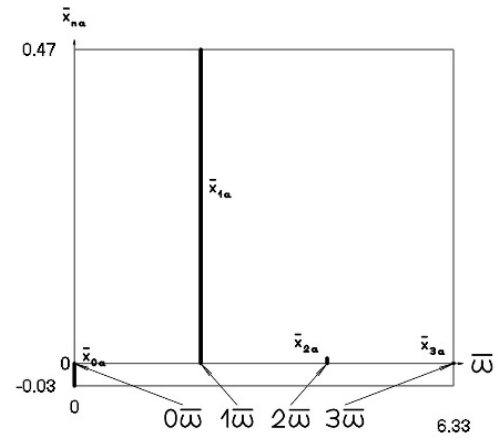

a) Displacement frequency characteristic

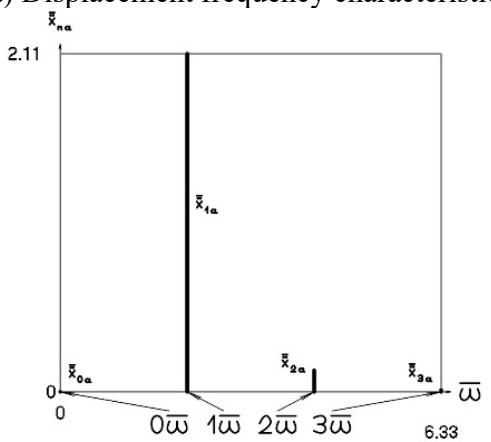

c) Acceleration frequency characteristic

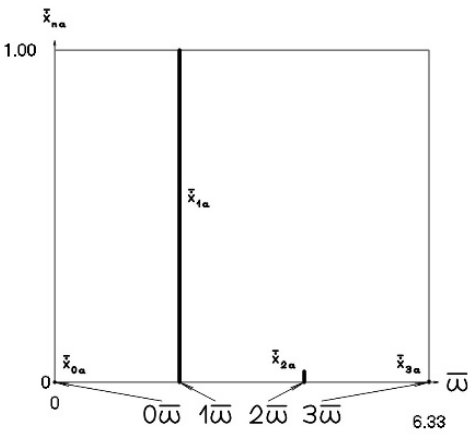

b) Velocity frequency characteristic

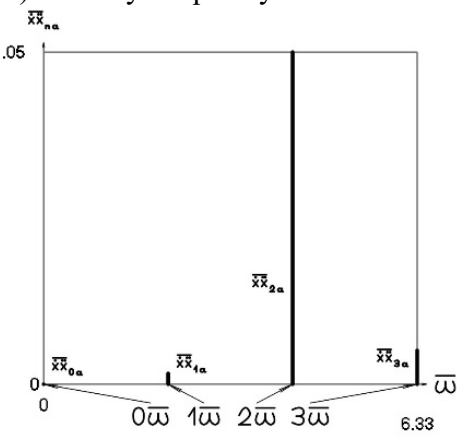

d) Velocity multiplied by acceleration frequency characteristic

Fig. 6. Amplitude frequency characteristics (constant part and first three harmonics) when $p^{2}=4, p_{0}^{2}=1$ 


\section{Conclusions}

General nonlinear model of the vibrator consists from two linear parts the coefficients of stiffness of the elastic element of which have different values and the positions of static equilibrium of the general system as well as positions of static equilibrium of both constituent parts are mutually equal. It is determined that the spectrum of eigenfrequencies of the general system is infinite and that values of frequencies do not depend from the amplitude of excitation. Vibrators of this type are effective, because in the resonance zones they do not have multivalued steady state regimes. The investigations were performed by using analytical - numerical methods and they revealed the qualities of the general system.

One period of motion of the conservative system is investigated. Results of investigation of dynamics of the conservative vibrating system for various parameters of the system are presented. Phase trajectories and amplitude frequency characteristics are used for representation of qualities of the system.

The presented analytical relationships and numerically obtained graphical relationships reveal the qualities of the system and enable to choose the desirable parameters of motions.

\section{References}

[1] Wedig W. V. New resonances and velocity jumps in nonlinear road-vehicle dynamics. Procedia IUTAM, Vol. 19, 2016, p. 209-218.

[2] Li T., Gourc E., Seguy S., Berlioz A. Dynamics of two vibro-impact nonlinear energy sinks in parallel under periodic and transient excitations. International Journal of Non-Linear Mechanics, Vol. 90, 2017, p. 100-110.

[3] Zaitsev V. A. Global asymptotic stabilization of periodic nonlinear systems with stable free dynamics. Systems and Control Letters, Vol. 91, 2016, p. 7-13.

[4] Dankowicz H., Fotsch E. On the analysis of chatter in mechanical systems with impacts. Procedia IUTAM, Vol. 20, 2017, p. 18-25.

[5] Spedicato S., Notarstefano G. An optimal control approach to the design of periodic orbits for mechanical systems with impacts. Nonlinear Analysis: Hybrid Systems, Vol. 23, 2017, p. 111-121.

[6] Li W., Wierschem N. E., Li X., Yang T. On the energy transfer mechanism of the single-sided vibro-impact nonlinear energy sink. Journal of Sound and Vibration, Vol. 437, 2018, p. 166-179.

[7] Marshall J. S. Modeling and sensitivity analysis of particle impact with a wall with integrated damping mechanisms. Powder Technology, Vol. 339, 2018, p. 17-24.

[8] Salahshoor E., Ebrahimi S., Zhang Y. Frequency analysis of a typical planar flexible multibody system with joint clearances. Mechanism and Machine Theory, Vol. 126, 2018, p. 429-456.

[9] Starossek U. Forced response of low-frequency pendulum mechanism. Mechanism and Machine Theory, Vol. 99, 2016, p. 207-216.

[10] Wang S., Hua L., Yang C., Zhang Y., Tan X. Nonlinear vibrations of a piecewise-linear quarter-car truck model by incremental harmonic balance method. Nonlinear Dynamics, Vol. 92, 2018, p. 1719-1732.

[11] Alevras P., Theodossiades S., Rahnejat H. On the dynamics of a nonlinear energy harvester with multiple resonant zones. Nonlinear Dynamics, Vol. 92, 2018, p. 1271-1286.

[12] Sinha A., Bharti S. K., Samantaray A. K., Chakraborty G., Bhattacharyya R. Sommerfeld effect in an oscillator with a reciprocating mass. Nonlinear Dynamics, Vol. 93, 2018, p. 1719-1739.

[13] Habib G., Cirillo G. I., Kerschen G. Isolated resonances and nonlinear damping. Nonlinear Dynamics, Vol. 93, 2018, p. 979-994.

[14] Ragulskis K., Ragulskis L. Vibroimpact mechanism in one separate case. Mathematical Models in Engineering, Vol. 5, Issue 2, 2019, p. 56-63. 

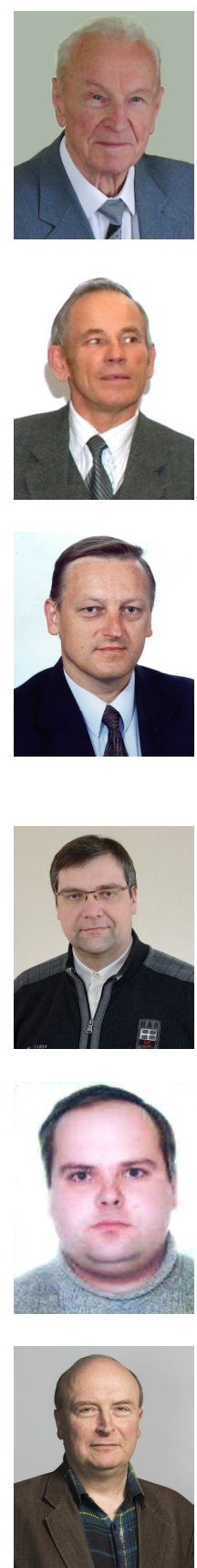

Kazimieras Ragulskis is a member of Academies of Sciences of the USSR (later of the Russian Academy of Sciences) and Lithuania. His research area is precise vibromechanics and vibroengineering, the basis of it are vibrations and waves in nonlinear dynamical systems. In this paper Kazimieras Ragulskis is responsible for conceptualization, formal analysis, investigation, methodology, supervision, validation, visualization, writing original draft preparation, writing - review and editing.

Bronislovas Spruogis is a Professor of the Department of Mobile Machinery and Railway Transport of the Faculty of Transport Engineering of Vilnius Gediminas Technical University. His main research interests are dynamics and design of mechanical and hydraulic systems. In this paper Bronislovas Spruogis is responsible for formal analysis, investigation, methodology, supervision, validation, visualization, writing - original draft preparation, writing - review and editing.

Marijonas Bogdevičius is Academician of the International Academy of Noosphere, Academician of the International Academy of Informatisation, Academician of the International Virtual Academy, Professor and Head of the Department of Mobile Machinery and Railway Transport of the Faculty of Transport Engineering of Vilnius Gediminas Technical University. His main research interests are dynamics and design of transport systems. In this paper Marijonas Bogdevičius is responsible for formal analysis, investigation, methodology, supervision, validation, visualization, writing - original draft preparation, writing - review and editing.

Arvydas Matuliauskas is a Lecturer of the Department of Mobile Machinery and Railway Transport of the Faculty of Transport Engineering of Vilnius Gediminas Technical University. His main research interests are dynamics and design of robots. In this paper Arvydas Matuliauskas is responsible for formal analysis, investigation, methodology, validation, visualization, writing - original draft preparation, writing - review and editing.

Vygantas Mištinas is a Lecturer of the Department of Mobile Machinery and Railway Transport of the Faculty of Transport Engineering of Vilnius Gediminas Technical University. His main research interests are dynamics and design of robots. In this paper Vygantas Mištinas is responsible for formal analysis, investigation, methodology, validation, visualization, writing - original draft preparation, writing - review and editing.

Liutauras Ragulskis is working as a research associate at Vytautas Magnus University. His research interests are numerical calculations of vibrating systems. In this paper Liutauras Ragulskis is responsible for visualization. 